\title{
The Importance of Methodological Rigor in Proof-of-Concept Clinical Trials: A Lesson from Hidradenitis Suppurativa
}

Journal of Investigative Dermatology (2020) $\mathbf{\square , ~} \mathbf{\square - \mathbf { n }}$; doi:10.1016/j.jid.2020.03.977

\section{TO THE EDITOR}

The methodological rigor and high standard of clinical trials give physicians the confidence that the therapy prescribed is well-tolerated, demonstrates clinical efficacy, and (within the restrictions set by the trial) is appropriate for the patient who presents to the clinic. Given that hidradenitis suppurativa (HS) has only one Food and Drug Administration-approved mAb to date (adalimumab), the plethora of ongoing clinical trials (van Straalen et al., 2018) provides solace that multiple equally (or hopefully more) efficacious therapeutic options will be available in the future. One of these new treatment options is bermekimab, a fully human antibody targeting IL- $1 \alpha$. Therefore, we read the phase 2 openlabel study of bermekimab in HS by Gottlieb et al. (2020) with great expectations. However, we have identified methodological concerns, which give a reason for pause and reflection.

The targeted aims of phase 2 studies often focus on addressing specific questions before the initiation of a complex, large, and expensive phase 3 clinical trial. The initial proof-ofconcept study by Kanni et al. (2018) demonstrated intravenous dosing of bermekimab as well-tolerated with significant changes in disease activity (60\% of patients achieving HS clinical response [HiSCR]) compared with placebo $(10 \%)$. The phase 2 study by Gottlieb et al. (2020) aimed to assess tolerability and clinical efficacy of a subcutaneous dose of bermekimab (400 mg weekly) with the number of patients achieving HiSCR at week 12 being the primary outcome of interest.
Clinical data collection and compliance with the core outcomes set

Although the HiSCR is considered the gold standard outcome measure for $\mathrm{HS}$, there is considerable discussion regarding the use of alternative measures because of issues with elevated placebo response rates, high interrater variability, and a lack of assessment of draining tunnels, which are a highly burdensome feature of HS (Frew et al., 2019). The historic initiative has defined a globally accepted core outcome set, which is recommended to be employed in the setting of HS clinical trials (Thorlacius et al., 2018) to aid comparison between different therapies. This includes data regarding Hurley staging, draining tunnels, and comorbidities as well as the presentation of demographic data to assess the external validity of trial participants (Thorlacius et al., 2018). Unfortunately, such data were not presented for this study (Gottlieb et al., 2020). The importance of capturing these data in early-stage proof-of-concept trials is manifest in light of the recent experience with IFX-1. IFX-1 is a complement (C5a) mAb, which demonstrated comparable clinical efficacy to adalimumab in the SHINE phase $2 \mathrm{~b}$ study (NCT03487276) (InflaRx, 2019) and met all secondary end points (including a significant reduction in pain and draining tunnels) but did not meet statistical significance compared with placebo. This was highly unexpected because of the high-response rate in an uncontrolled trial (NCT03001622) (Giamarellos-Bourboulis et al., 2020). Although the HiSCR itself as an outcome measure may have

Abbreviations: HiSCR, HS clinical response; HS, hidradenitis suppurativa; LOCF, last observation carried forward

Accepted manuscript published online XXX; corrected proof published online XXX

() 2020 The Authors. Published by Elsevier, Inc. on behalf of the Society for Investigative Dermatology. contributed to the high placebo response rates $(47 \%)$ (Frew et al., 2019), the fact that the data regarding efficacy against draining tunnels were captured (and were shown to be significant against placebo) (InflaRx, 2019) highlights the importance of capturing all relevant clinical variables in proof-of-concept clinical trials, especially in uncontrolled trials. The emerging evidence that specific lesions such as draining tunnels can be associated with treatment response emphasizes the need for breadth and consistency in core outcomes in HS clinical trials.

\section{Variations in administered doses of bermekimab}

A major concern regarding methodology in this study (Gottlieb et al., 2020) was the unspecified number of patients who started the trial with a $200 \mathrm{mg}$ subcutaneous dose of bermekimab weekly (as outlined in the methods) but were increased to a $400 \mathrm{mg}$ subcutaneous weekly dose at an unspecified time point, that is, at the patient's next scheduled visit. This deviation from the original protocol (or protocol implied in the manuscript) raises significant concern that this paper presents results of a cohort of not only patients administered different doses of bermekimab (200 mg weekly vs. 400 mg weekly) but also participants who have received different doses for different lengths of time before assessment of the primary outcome. The lack of consideration of this ad-hoc dose alteration either through exclusion or statistical methods brings into question the validity of the study results.

Imputation methods for participant dropout before primary end point Gottlieb et al. (2020) describe the use of last observation carried forward (LOCF) as their imputation method for participants who withdrew from the 
KR van Straalen and JW Frew

Methodological Rigor: A Lesson from HS study or were lost to follow-up $(n=9)$. LOCF has been used in a number of HS-related studies (Casseres et al., 2020; Zouboulis et al., 2019) as an imputation method. However, its use is not recommended in circumstances where dropout can occur owing to a lack of efficacy because this leads to an increased risk of positively biasing treatment response rates. Verifying the findings of the LOCF analysis with a nonresponder imputation, regarding all withdrawals as secondary to lack of treatment efficacy, would increase the validity of the data (National Research Council, 2010). Given the moderate rates of achieving clinical outcomes in HS studies $(50-60 \%)$, the suitability of LOCF analysis in HS studies requires serious consideration. The flaws of LOCF analysis in HS are clearly illustrated by the open-label study of secukinumab in HS (Casseres et al., 2020), where 13 of 20 patients $(65 \%)$ achieved HiSCR at week 12. Using nonresponder imputation, the number of participants achieving HiSCR is revised down to 11 of 20 (55\%). Regarding the data presented by Gottlieb et al. (2020), when conservatively only applying nonresponder imputation to those participants who were lost to follow-up ( $\mathrm{n}=3$, all in group $B$ ) or elected to discontinue without an alternate reason $(n=2$, one in group $\mathrm{A}$ and one in group $\mathrm{B}$ ), the revised rates of achieving HiSCR would equate to $54.2 \%(n=13)$ in group $A$ and $38.9 \%(n=7)$ in group B. In order to refute this nonresponder imputation, an explanation as to the HiSCR status of participants upon withdrawal would be required and would be a highly useful addition to the results of all future HS clinical trials.
In conclusion, high-quality methodologies in proof-of-concept uncontrolled clinical trials are vital to accurately assess clinical efficacy. The results of this phase 2 trial with bermekimab need to be interpreted with care because they are subject to several methodological issues. Pause and consideration of the issues discussed should be taken before the development of phase 3 clinical trials for bermekimab in $\mathrm{HS}$.

\section{Data availability statement}

No datasets were generated or analyzed during this study.

\section{ORCIDs}

Kelsey R. van Straalen: http://orcid.org/00000003-3305-3814

John W. Frew: http://orcid.org/0000-0001-5 042-3632

\section{CONFLICT OF INTEREST}

The authors state no conflict of interest.

\section{AUTHOR CONTRIBUTIONS}

Conceptualization: KRVS, JWF; Investigation: KRVS, JWF; Writing - Original Draft Preparation: KRVS, JWF; Writing - Review and Editing: KRVS, JWF

\section{Kelsey R. van Straalen ${ }^{1}$ and John W. Frew ${ }^{2, *}$}

${ }^{1}$ Department of Dermatology, Erasmus University Medical Center, Rotterdam, The Netherlands; and ${ }^{2}$ Laboratory of Investigative Dermatology, Rockefeller University, New York, USA

*Corresponding author e-mail: jfrew@ rockefeller.edu

\section{REFERENCES}

Casseres RG, Prussick L, Zancanaro P, Rothstein B, Joshipura D, Saraiya A, et al. Secukinumab in the treatment of moderate to severe hidradenitis suppurativa: results of an open-label trial. J Am Acad Dermatol 2020;82:1524-6.

Frew JW, Jiang CS, Singh N, Grand D, Navrazhina K, Vaughan $R$, et al. Clinical response rates, placebo response rates and significantly associated covariates are depen- dent upon choice of outcome measure in hidradenitis suppurativa: a post-hoc analysis of pioneer 1 and 2 individual patient data. J Am Acad Dermatol 2020;82:1150-7.

Giamarellos-Bourboulis EJ, Argyropoulou M, Kanni T, Spyridopoulos T, Otto I, Zenker O, et al. Clinical efficacy of complement c5a inhibition by ifx- 1 in hidradenitis suppurativa: an open-label single-arm trial in patients not eligible for adalimumab [e-pub ahead of print]. Br J Dermatol 2020. https:// doi.org/10.1111/bjd.18877 (accessed 10 February 2020)

Gottlieb A, Natsis NE, Kerdel F, Forman S, Gonzalez E, Jimenez G, et al. A phase II, openlabel study of bermekimab in patients with hidradenitis suppurativa shows resolution of inflammatory lesions and pain [e-pub ahead of print]. J Invest Dermatol 2020. https://doi.org/ 10.1016/j.jid.2019.10.024 (accessed 10 February 2020).

InflaRx. 07-2019-InflaRx reports additional analysis of the SHINE Phase IIb results for IFX-1 in hidradenitis suppurativa. 2019. accessed, https:// www.inflarx.de/Home/Investors/Press-Releases/ 07-2019-InflaRx-Reports-Additional-Analysis-ofthe-SHINE-Phase-IIb-Results-for-IFX-1-in-Hidradenitis-Suppurativa-.html. (accessed February 10, 2020).

Kanni T, Argyropoulou M, Spyridopoulos T, Pistiki A, Stecher M, Dinarello CA, et al. MABp1 targeting IL-1 $\alpha$ for moderate to severe hidradenitis suppurativa not eligible for adalimumab: a randomized study. J Invest Dermatol 2018;138:795-801.

National Research Council (US) panel on handling missing data in clinical trials. The prevention and treatment of missing data in clinical trials. Washington, DC: National Academies Press; 2010.

Thorlacius L, Ingram JR, Villumsen B, Esmann S, Kirby JS, Gottlieb AB, et al. A core domain set for hidradenitis suppurativa trial outcomes: an international Delphi process. $\mathrm{Br} J$ Dermatol 2018;179:642-50

van Straalen KR, Schneider-Burrus S, Prens EP. Current and future treatment of hidradenitis suppurativa [e-pub ahead of print]. $\mathrm{Br} J \mathrm{Der}$ matol 2018. https://doi.org/10.1111/bjd.16768 (accessed 10 February 2020).

Zouboulis CC, Okun MM, Prens EP, Gniadecki R, Foley PA, Lynde C, et al. Long-term adalimumab efficacy in patients with moderate-tosevere hidradenitis suppurativa/acne inversa: 3 -year results of a phase 3 open-label extension study. J Am Acad Dermatol 2019;80:60-69.e2. 